BMJ Open

Diabetes

Research

\& Care

\title{
Adipokines in early and mid-pregnancy and subsequent risk of gestational diabetes: a longitudinal study in a multiracial cohort
}

Ellen C Francis (D) , ${ }^{1,2}$ Mengying Li (D) , ${ }^{2}$ Stefanie N Hinkle, ${ }^{2}$ Yaqi Cao, ${ }^{3}$ Jinbo Chen, ${ }^{3}$ Jing Wu, ${ }^{4}$ Yeyi Zhu, ${ }^{5,6}$ Haiming Cao, ${ }^{7}$ Karen Kemper, ${ }^{8}$ Lior Rennert, ${ }^{8}$ Joel Williams, ${ }^{8}$ Michael Y Tsai, ${ }^{9}$ Liwei Chen, ${ }^{10}$ Cuilin Zhang ${ }^{2}$

To cite: Francis EC, Li M, Hinkle SN, et al. Adipokines in early and mid-pregnancy and subsequent risk of gestational diabetes: a longitudinal study in a multiracial cohort. BMJ Open Diab Res Care 2020;8:e001333. doi:10.1136/ bmjdrc-2020-001333

- Additional material is published online only. To view please visit the journal online (http://dx.doi.org/10.1136/ bmjdrc-2020-001333).

Received 5 March 2020 Revised 7 May 2020 Accepted 18 May 2020
Check for updates

\section{(C) Author(s) (or their} employer(s)) 2020. Re-use permitted under CC BY-NC. No commercial re-use. See rights and permissions. Published by BMJ.

For numbered affiliations see end of article.

Correspondence to Dr Cuilin Zhang; zhangcu@mail.nih.gov

\section{ABSTRACT}

Introduction Several adipokines are implicated in the pathophysiology of gestational diabetes mellitus (GDM), however, longitudinal data in early pregnancy on many adipokines are lacking. We prospectively investigated the association of a panel of adipokines in early and midpregnancy with GDM risk.

Research design and methods Within the National Institute of Child Health and Human Development (NICHD) Fetal Growth Studies-Singletons cohort ( $n=2802)$, a panel of 10 adipokines (plasma fatty acid binding protein-4 (FABP4), chemerin, interleukin-6 (IL-6), leptin, soluble leptin receptor (SOB-R), adiponectin, omentin-1, vaspin, and retinol binding protein-4) were measured at gestational weeks (GWs) 10-14, 15-26, 23-31, and 33-39 among 107 GDM cases (ascertained on average at GW 27) and 214 non-GDM controls. Conditional logistic regression was used to estimate ORs of each adipokine and GDM, controlling for known GDM risk factors including pre-pregnancy body mass index. Results Throughout pregnancy changes in chemerin, sOB-R, adiponectin, and high-molecular-weight adiponectin (HMWadiponectin) concentrations from 10-14 to 15-26 GWs were significantly different among GDM cases compared with non-GDM controls. In early and mid-pregnancy, FABP4, chemerin, IL-6 and leptin were positively associated with increased GDM risk. For instance, at 10-14 GWs, the OR comparing the highest versus lowest quartile (ORQ4-Q1) of FABP4 was 3.79 ( $(95 \% \mathrm{Cl} 1.63$ to 8.85). In contrast, in both early and mid-pregnancy adiponectin (eg, ORQ4-Q1 0.14 $(0.05,0.34)$ during 10-14 GWs) and SOB-R (ORQ4-Q1 0.23 $(0.11,0.50)$ during $10-14 \mathrm{GWs})$ were inversely related to GDM risk. At 10-14 GWs a model that included conventional GDM risk factors and FABP4, chemerin, SOB-R, and HMWadiponectin improved the estimated prediction (area under the curve) from $0.71(95 \% \mathrm{Cl} 0.66$ to 0.77$)$ to $0.77(95 \% \mathrm{Cl}$ 0.72 to 0.82 ).

Conclusions A panel of understudied adipokines including FABP4, chemerin, and SOB-R may be implicated in the pathogenesis of GDM with significant associations detected approximately 10-18 weeks before typical GDM screening.

\section{INTRODUCTION}

Gestational diabetes mellitus (GDM), a common pregnancy complication marked by

\section{Significance of this study}

What is already known about this subject?

- Some adipokines have been implicated in the pathophysiology of gestational diabetes mellitus (GDM), however, longitudinal adipokine data beginning in the first trimester of human pregnancy are lacking and this impedes our understanding of their association with GDM risk.

What are the new findings?

- We found that as early as 10-14 gestational weeks, understudied adipokines such as fatty acid binding protein-4 and chemerin were significantly associated with risk of developing GDM.

- Inclusion of adipokines in a predictive model improved the area under the curve value beyond conventional risk factors and glucose.

- Adipokines were prospectively correlated with cardiometabolic markers.

\section{How might these results change the focus of} research or clinical practice?

- Findings from this study have the potential to improve our understanding of the pathogenesis of GDM and inform future efforts in disease prediction.

glucose intolerance resulting from insufficient insulin secretion relative to pregnancy induced insulin resistance, ${ }^{1}$ is associated with both short-term and long-term adverse maternal and offspring health outcomes. ${ }^{2}$ In the USA, GDM is typically screened for between 24 and 28 gestational weeks (GWs), leaving only the third trimester for intervention or treatment. Prediagnostic markers of GDM risk in early pregnancy are warranted and could have pathophysiological relevance and inform efforts into earlier diagnoses and prevention.

Adipokines mediate crosstalk between adipose tissue and key organs and tissues involved in glucose homeostasis, such as the 
skeletal muscle, liver, and pancreas. ${ }^{3}$ Adipokines also modulate multiple pathways for insulin signaling and secretion, lipid metabolism, inflammatory responses, and endothelial function. ${ }^{3}$ Among women with pregnancies complicated by GDM, a disruption in these regulatory pathways is observed, ${ }^{4}$ suggesting that adipokines may play a role in the etiology of GDM.

Commonly studied adipokines such as leptin and adiponectin have been repeatedly associated with GDM risk, indicating their potential roles in the pathophysiology of GDM. ${ }^{35}$ Accumulating data from animal and in vitro studies suggested that other adipokines such as fatty acid binding protein-4 (FABP4), chemerin, soluble leptin receptor (sOB-R), omentin-1, vaspin, and retinol binding protein-4 (RBP4) are implicated in insulin signaling ${ }^{3}$ and insulin sensitivity, ${ }^{6}$ however, the lack of human prospective studies focused on these adipokines and GDM risk with early to mid-pregnancy data have hindered our inference into their pathophysiological relevance ${ }^{7-15}$ Further, there is a lack of data from studies among diverse racial/ ethnic populations, with the majority conducted among Asian women. ${ }^{8-15}$ Only one study was conducted among a diverse population, however, it included a small number of women with GDM $(\mathrm{n}=48){ }^{7}$

In the current study, we investigated the longitudinal changes in a panel of 10 adipokines (ie, FABP4, chemerin, IL-6, leptin, sOB-R, adiponectin and highmolecular-weight adiponectin (HMW-adiponectin), omentin-1, vaspin, and RBP4) throughout pregnancy, and prospectively examined concentrations of them in early to mid-pregnancy (before the diagnosis of GDM) in association with the risk of GDM. To better understand the pathophysiology of any observed association between adipokines and GDM, we further investigated the prospective correlations of levels of adipokines in the first trimester with second trimester levels of clinical cardiometabolic biomarkers (ie, glucose, insulin, and lipids) prior to GDM diagnosis.

\section{RESEARCH DESIGN AND METHODS}

\section{Study population and design}

This study used data from a nested case-control study of GDM within the prospective Eunice Kennedy Shriver National Institute of Child Health and Human Development (NICHD) Fetal Growth Studies-Singletons cohort ${ }^{16}$ which enrolled 2802 pregnant women between GWs 8 and 13 from 12 US clinical centers from 2009 to 2013. Biospecimens, questionnaires, and maternal anthropometric data were collected longitudinally throughout pregnancy. Information on prenatal care and delivery were abstracted from medical records following delivery. Blood samples were longitudinally collected at four study visits following a standardized protocol; 10-14 GWs, fasting sample at 15-26 GWs, 23-31 GWs, and 33-39 GWs. The study was approved by the institutional review boards of all participating institutions. All participants provided written, informed consent. All methods were performed in accordance with the relevant guidelines and regulations.

A total of 107 GDM cases were identified and matched to 214 controls on a 1:2 ratio based on maternal age ( \pm 2 years), race/ethnicity, and GW of blood collection ( \pm 2 weeks). ${ }^{17}$ Women were classified with GDM if their oral glucose tolerance test results met the CarpenterCoustan criteria of at least two diagnostic plasma glucose measurements at or above the defined thresholds (fasting $5.3 \mathrm{mmol} / \mathrm{L}, 1$ hour $10.0 \mathrm{mmol} / \mathrm{L}, 2$ hours $8.6 \mathrm{mmol} / \mathrm{L}$, 3 hours $7.8 \mathrm{mmol} / \mathrm{L})^{18} 19$ or if medication-treated GDM was reported on their medical record.

\section{Biomarker assessment}

For the blood samples collected before the diagnosis of GDM (10-14 and 15-26 GWs) biomarkers were assayed among all cases and controls. For blood samples collected at 23-31 and 33-39 GWs, biomarkers were assayed in all cases $(\mathrm{n}=107)$ and one randomly selected control $(\mathrm{n}=107)$.

Assay method and details on coefficients of variations (CV) of plasma adipokines (FABP4, chemerin, IL-6, leptin, sOB-R, adiponectin, HMW-adiponectin, omentin-1, vaspin, and RBP4), and plasma glucose metabolism and cardiometabolic biomarkers (ie, glucose, insulin, high-sensitivity c-reactive protein (hsCRP), total cholesterol, high-density lipoprotein (HDL) cholesterol, and triglycerides) can be found in online supplementary table 1 . All CVs were $\leq 18.0 \%$.

\section{Covariates}

Maternal sociodemographic characteristics were collected from detailed questionnaires at enrollment. Risk factors for GDM selected a priori as covariates included: family history of diabetes (yes/no), nulliparity (yes/no), and prepregnancy body mass index $\left(\mathrm{BMI} ; \mathrm{kg} / \mathrm{m}^{2}\right)$. Prepregnancy BMI was calculated based on self-reported weight and measured height during enrollment. Self-reported prepregnancy weight was highly correlated with weight measured by study personnel during the enrollment visit $(r=0.97) .{ }^{20}$ All models were adjusted for the continuous measures of the case/control matching factors of age and GW at blood draw, which were matched only within a range.

\section{Statistical analyses}

Differences in participant baseline characteristics by GDM status were assessed using mixed-effect linear regression for continuous variables and conditional logistic regression for categorical variables, accounting for matched case-control pairs.

\section{Adipokine profiles throughout pregnancy}

Median concentrations of adipokines among cases and controls were plotted by study visit, and differences in adipokine values by GDM status at each visit were tested using mixed-effect linear regression models with log-transformed levels of adipokines to account for the skewed distribution. We further tested to see if changes in 
adipokine concentrations from 10-14 GWs to 15-26 GWs (before GDM screening) differed by GDM status after adjusting for enrollment (10-14 GWs) concentrations. For presentation of the results, adipokine concentrations were back transformed to the original scale.

\section{Prospective association between adipokines and GDM risk}

Prepregnancy BMI partially reflects total subcutaneous and visceral adipose tissue, both of which are secretory sources of adipokines and contribute to their circulating concentrations. ${ }^{321}$ Adjusting for prepregnancy BMI as a risk factor for GDM results in a model that at least partially controls for the source of the exposure of interest. Models including prepregnancy BMI would therefore mostly reflect adipokine concentrations secreted primarily from other sources (eg, hepatic, placental). To address this issue, the current analysis examined multivariable models with, and without, adjustment for prepregnancy BMI. Multivariable conditional logistic regression models adjusting for the above-listed covariates were fitted to assess the associations of each adipokine at 10-14 GWs and 15-26 GWs with subsequent GDM risk. To ensure that biomarker measurements preceded the diagnosis of GDM, we excluded from the final analysis GDM cases diagnosed prior to blood sample collection ( $\mathrm{n}=1$ at $10-14$ GWs; n=5 at 15-26 GWs). We further excluded participants with Hemoglobin A1c $\geq 6.5 \%(48 \mathrm{mmol} / \mathrm{mol})$ at enrollment $(n=3)$, as this is an indicator of undiagnosed overt type 2 diabetes. ${ }^{22}$

The OR of GDM was estimated by quartiles of adipokine concentrations based on the distribution among controls, with the lowest quartile as the reference group. Tests of linear trends were performed modeling the median value of each quartile as a continuous variable. Tests for non-linear associations were performed using restricted cubic splines. ${ }^{23}$ We further explored effect modification in the association between adipokines and GDM by examining interactions between adipokines and maternal race/ethnicity (non-Hispanic white, nonHispanic black, Hispanic, or Asian/Pacific Islander).

\section{Prospective correlations between adipokines and glucose metabolism and cardiometabolic biomarkers}

To examine the prospective correlation of adipokines and glucose metabolism and cardiometabolic biomarkers, we calculated partial Spearman's correlation coefficients adjusted for covariates among non-GDM controls. NonGDM controls were selected to more closely represent the correlation among normal pregnant women without underlying disruptions in insulin resistance. Spearman's correlations were calculated for each adipokine at 10-14 GWs with glucose metabolism (fasting plasma glucose, insulin and the homeostatic model assessment of insulin resistance (HOMA-IR) ${ }^{24}$ ) and cardiometabolic markers (hsCRP, total cholesterol, HDL cholesterol, low-density lipoprotein (LDL), cholesterol, and triglycerides) at the subsequent visit (15-26 GWs).

\section{Exploratory analysis of an adipokine score and GDM risk}

An exploratory analysis was carried out to assess the risk of GDM associated with combined categories of adipokines that were independently associated with GDM at 10-14 GWs and 15-26 GWs using an adipokine score. ${ }^{25} \mathrm{~A}$ dichotomous high-risk variable was created by setting cutoffs for each adipokine at the lowest quartile if levels were lower among cases, and at the highest quartile if levels were higher among cases. Each high-risk variable was multiplied by their respective coefficient generated from a conditional logistic regression model that included all adipokines associated with GDM in multivariable models and previously listed covariates (excluding prepregnancy BMI). The weighted score was generated by taking the sum of each adipokine high-risk variable and categorizing it, with a score of zero set as the reference category, and the remaining categories based on tertiles from the distribution of non-zero scores within the controls. The association of GDM with categories of the adipokine score were estimated by multivariable conditional logistic regression models adjusting for the above-listed covariates.

\section{Comparison of adipokines for GDM prediction}

At 10-14 GWs and 15-26 GWs, we compared the predictive performance of a model that included conventional GDM risk factors (ie, maternal age, race/ethnicity, family history of diabetes, and plasma random glucose (10-14 GWs), or fasting plasma glucose (15-26 GWs)) to a model that included conventional GDM risk factors and individual adipokines. Receiver operating characteristic curves were plotted and the areas under the curves (AUCs) were calculated ${ }^{26}$ based on analyses that modeled the data as if they were generated from a twophase design. ${ }^{27}$ Bootstrapping was applied to correct bias due to potential model overfitting in estimates of statistical measures for overall predictive performance for each model. ${ }^{28}{ }^{29}$ Continuous adipokines were categorized as quartiles based on the distribution among controls. A final risk prediction model was developed by sequentially adding adipokines until the AUC stabilized. ${ }^{30}$ Prediction analyses were implemented in R V.3.5.1. All other analyses were implemented using SAS V.9.4 (SAS Institute, Cary, North Carolina, USA), with $\alpha<0.05$ as the level of significance.

\section{RESULTS}

\section{Characteristics of study participants and adipokine profiles} throughout pregnancy by GDM status

Participant characteristics are presented in table 1.

Women who developed GDM were more likely to have a family history of diabetes and have a higher prepregnancy BMI compared with non-GDM controls. At all four study visits, concentrations of FABP4, chemerin, and IL-6 were significantly higher among GDM cases, and concentrations of sOB-R were lower compared with controls (all $\mathrm{p}$ values $<0.05$ ) (figure 1). Prior to GDM diagnosis (10-14 GWs and 15-26 GWs), leptin was higher, whereas 
Table 1 Characteristics among women with GDM and their matched controls, the NICHD Fetal Growth StudySingletons cohort

\begin{tabular}{|c|c|c|c|}
\hline & GDM cases & Controls & P value* \\
\hline Age (years) & $30.5 \pm 5.7$ & $30.4 \pm 5.4$ & \\
\hline \multicolumn{4}{|l|}{ Race/ethnicity } \\
\hline Non-Hispanic white & $25(23.4)$ & $50(23.4)$ & \\
\hline Non-Hispanic black & $15(14.0)$ & $30(14.0)$ & \\
\hline Hispanic & $41(38.3)$ & $82(38.3)$ & \\
\hline $\begin{array}{l}\text { Asian/Pacific } \\
\text { Islander }\end{array}$ & $26(24.3)$ & $52(24.3)$ & \\
\hline Education & & & 0.18 \\
\hline $\begin{array}{l}\text { Less than high } \\
\text { school }\end{array}$ & $17(15.9)$ & $26(12.1)$ & \\
\hline $\begin{array}{l}\text { High school } \\
\text { graduate or } \\
\text { equivalent }\end{array}$ & $15(14.0)$ & $23(10.8)$ & \\
\hline $\begin{array}{l}\text { More than high } \\
\text { school }\end{array}$ & $75(70.1)$ & $165(77.1)$ & \\
\hline \multicolumn{4}{|l|}{ Insurance } \\
\hline $\begin{array}{l}\text { Private or managed } \\
\text { care }\end{array}$ & $68(63.5)$ & $143(66.8)$ & \\
\hline $\begin{array}{l}\text { Medicaid, self-pay, } \\
\text { other }\end{array}$ & 39 (36.5) & $71(33.2)$ & 0.50 \\
\hline \multicolumn{4}{|l|}{ Marital status } \\
\hline Never married & $11(10.3)$ & 35 (16.4) & \\
\hline $\begin{array}{l}\text { Married/living with a } \\
\text { partner }\end{array}$ & $92(86.0)$ & $167(78.0)$ & \\
\hline Divorced/separated & $4(3.7)$ & $12(5.6)$ & 0.12 \\
\hline Nulliparity & $48(44.9)$ & $96(44.9)$ & 1.00 \\
\hline $\begin{array}{l}\text { Family history of } \\
\text { diabetes }\end{array}$ & $40(37.4)$ & $48(22.4)$ & 0.001 \\
\hline $\begin{array}{l}\text { Prepregnancy BMI } \\
\left(\mathrm{kg} / \mathrm{m}^{2}\right)\end{array}$ & $28.2 \pm 6.4$ & $25.6 \pm 5.3$ & $<0.001$ \\
\hline$<25.0$ & $37(34.6)$ & $123(58.0)$ & \\
\hline $25.0-29.9$ & $35(32.7)$ & $56(26.2)$ & \\
\hline $30.0-34.9$ & $20(18.7)$ & $17(7.9)$ & \\
\hline $35.0-44.9$ & $15(14.0)$ & $16(7.5)$ & \\
\hline Unknown/missing & & $2(0.9)$ & $<0.001$ \\
\hline $\begin{array}{l}\text { Smoking } 6 \text { months } \\
\text { before pregnancy }\end{array}$ & $4(3.7)$ & $1(0.5)$ & 0.06 \\
\hline $\begin{array}{l}\text { Alcohol consumption } \\
3 \text { months before } \\
\text { pregnancy }\end{array}$ & $61(57.0)$ & $137(64.0)$ & 0.22 \\
\hline
\end{tabular}

Data are presented as $n(\%)$ for categorical variables and mean (SD) for continuous variables

${ }^{*} \mathrm{P}$ values for differences between case and control subjects were obtained by mixed-effect linear regression models for continuous variables and binomial/multinomial logistic regression with generalized estimating equations for binary/ multilevel categorical variables, accounting for matched case-control pairs. $\mathrm{P}$ values are not shown for matching variables (age and race/ethnicity).

BMI, body mass index; GDM, gestational diabetes mellitus;

NICHD, National Institute of Child Health and Human Development. adiponectin and HMW-adiponectin were significantly lower among cases than controls (all $\mathrm{p}$ values $<0.05$ ). Further, changes in concentrations of chemerin, sOB-R, adiponectin, and HMW-adiponectin from 10-14 GWs to 15-26 GWs were significantly different between GDM cases and controls.

\section{Adipokines in relation to subsequent GDM risk}

The associations of adipokines measured at 10-14 GWs and 15-26 GWs with subsequent GDM risk are presented in table 2.

After adjustment for covariates other than prepregnancy BMI, at 10-14 GWs, FABP4, chemerin, IL-6, and leptin concentrations were significantly and positively associated with GDM risk, whereas sOB-R and adiponectin concentrations were significantly and inversely associated with GDM risk ( $p$ for linear trend all $<0.01$ ). For instance, compared with the lowest quartile, the highest quartile of sOB-R was associated with a $76 \%$ reduction in GDM risk at $10-14 \mathrm{GWs}(\mathrm{OR}=0.24 ; 95 \% \mathrm{CI} 0.11$ to 0.52$)$. At 15-26 GWs, in general, similar results were observed. Omentin-1, vaspin, or RBP4 were not significantly related to GDM risk. After further adjustment for prepregnancy BMI, most associations remained significant, although slightly attenuated (online supplementary table 2). Further, the associations did not differ materially by race/ethnicity.

Our exploratory analysis of the joint effects of multiple adipokines resulted in a weighted adipokine score including chemerin, FABP4, IL-6, leptin, sOB-R, and HMW-adiponectin (high-risk cut-off values and weights can be found in online supplementary table 3). After adjusting for covariates other than prepregnancy BMI, at 10-14 GWs, a score in the third tertile was associated with increased GDM risk (OR=7.51;95\% CI 2.87 to 19.66) compared with a score of zero (online supplementary table 3). At 15-26 GWs scores in both the second tertile (OR=6.32; 95\% CI 1.50 to 26.67) and third tertile $(\mathrm{OR}=14.87 ; 95 \mathrm{CI} 4.02$ to 55.02$)$ were associated with an increased GDM risk compared with a score of zero.

\section{Prospective correlation between adipokines and glucose metabolism and cardiometabolic biomarkers}

At 10-14 GWs, IL-6 and leptin were generally positively related to subsequent fasting glucose metabolism markers at 15-26 GWs, while sOB-R, adiponectin, HWMadiponectin, and omentin-1 were inversely related to glucose metabolism markers (table 3).

Further, FABP4, chemerin, and leptin were positively correlated, while sOB-R and omentin-1 were inversely associated with hsCRP. sOB-R, adiponectin, and omentin-1 were generally correlated with a favorable lipid profile, while chemerin and RBP4 were generally associated with a worse lipid profile.

\section{Comparison of adipokines for GDM prediction}

The estimated prediction accuracy for the different models (conventional risk factors or conventional risk 

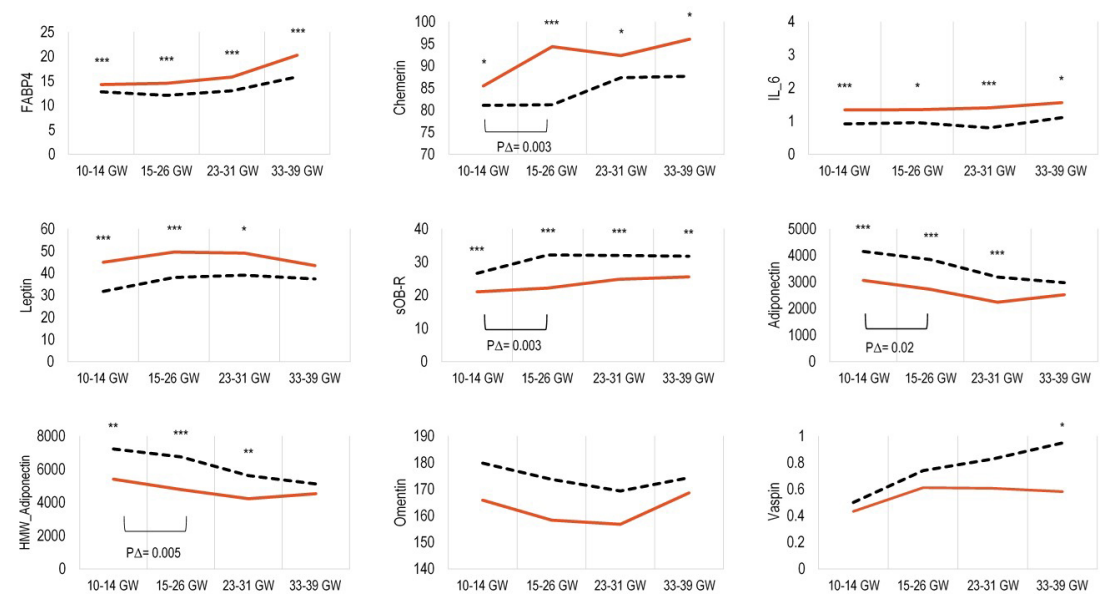

Figure 1 Median concentrations of adipokines, and longitudinal change from 10-14 GWs to 15-26 GWs expressed as $\mathrm{ng} / \mathrm{mL}$ (IL-6 expressed as pg/mL) according to gestational age intervals among women with GDM (solid red line) and their matched control subjects (dashed black line). ${ }^{*} \mathrm{P}<0.05$; ${ }^{* *} \mathrm{p}<0.01$; ${ }^{* * *} \mathrm{p}<0.001$ for case-control comparisons obtained by mixed-effect linear regression models accounting for matched case-control pairs at each gestational age interval. $P \Delta=p<0.05$ for casecontrol comparison of change in adipokine concentrations from 10-14 GWs to 15-26 GWs obtained by mixed-effect linear regression models accounting for matched case-control pairs. FABP-4, fatty acid binding protein 4; GDM, gestational diabetes mellitus; GW, gestational week; HMW-adiponectin, high-molecularweight-adiponectin; IL-6, interleukin-6; sOB-R, soluble leptin receptor.

factors plus adipokines) can be found in online supplementary table 4. At 10-14 GWs a final model that included the conventional GDM risk factors and FABP4, chemerin, sOB-R, and HMW-adiponectin improved the estimated AUC from 0.71 (95\% CI 0.66 to 0.77 ) to 0.77 (95\% CI 0.72 to 0.82 ) (online supplementary figure 1 ). At 15-26 GWs the final model with the same adipokines plus conventional GDM risk factors improved the estimated AUC from 0.75 (95\% CI 0.69 to 0.80$)$ to $0.82(95 \%$ CI 0.77 to 0.87 ) (online supplementary figure 1 )

\section{DISCUSSION}

In this longitudinal study, we prospectively and comprehensively evaluated concentrations of a panel of adipokines over the course of pregnancy and their associations with GDM risk. Beginning in early pregnancy, levels and trajectories of several adipokines differed by GDM status. Among this racially/ethnically diverse sample of US women, during early and mid-pregnancy higher levels of FABP4, chemerin, IL-6, and leptin were significantly associated with increased GDM risk, whereas higher levels of sOB-R, and adiponectin were significantly associated with reduced GDM risk. These adipokines were significantly and prospectively associated with glucose metabolism and cardiometabolic biomarkers, indicating that dysregulation in adipokines may disrupt glucose homeostatic processes underlying increased risk of GDM. To note, we found that many associations between adipokines and risk of GDM remained statistically significant after adjusting for prepregnancy BMI, which is a risk factor for hyperglycemia. However, the magnitude of the association was attenuated after adjusting for prepregnancy BMI indicating that some of the effect of adipokines on hyperglycemia operates through adiposity and thus may not be independent of obesity status.

FABP4 is a binding chaperon that regulates transport of lipid-based molecules. ${ }^{31}$ In the present study, higher concentrations of FABP4 in early and mid-pregnancy were significantly associated with increased GDM risk. Throughout pregnancy FABP4 levels were consistently higher among women who developed GDM compared with controls. Prospective studies of FABP4 and GDM are limited ${ }^{825}$ with the majority of previous studies being cross-sectional or retrospective. ${ }^{32}$ In a study among 1150 Chinese women (GDM cases n=135), FABP4 at $\sim 6$ GWs was positively associated with subsequent risk of developing GDM. ${ }^{8}$ However, in a study among 123 Australian women who were mostly (84\%) Caucasian, and had a prior GDM pregnancy, there were no differences in FABP4 levels at approximately $14 \mathrm{GWs}$ or $28 \mathrm{GWs}$ between women with and without recurrent GDM. ${ }^{25}$ Our findings add to the limited prospective data by demonstrating that FABP4 is associated with GDM risk among a diverse sample of pregnant women, and that higher concentrations of FABP4 among women with GDM persisted throughout pregnancy.

Although the precise molecular mechanisms are yet to be discovered, in vitro and animal models have implicated FABP4 in multiple pathways relevant to the pathogenesis of GDM. FABP4 promotes lipolysis, regulates fatty acid uptake and inflammation, and has been implicated in impairing insulin action and upregulating hepatic gluconeogenesis. ${ }^{33}$ These findings are in line with our assessment of the correlations between FABP4 and cardiometabolic markers. Specifically, we observed that among normoglycemic women FABP4 was positively correlated with subsequent fasting levels of glucose, 
Table 2 Adjusted OR $(95 \% \mathrm{Cl})$ for the associations of GDM risk with quartiles of adipokines at gestational weeks 10-14 and 15-26

\begin{tabular}{|c|c|c|c|c|c|c|}
\hline & \multirow{2}{*}{$\begin{array}{l}\text { GDM } \\
\text { cases (n) }\end{array}$} & \multirow{2}{*}{$\begin{array}{l}\text { Controls } \\
\text { (n) }\end{array}$} & \multicolumn{2}{|c|}{ 10-14 GWs multivariable } & \multicolumn{2}{|c|}{ 15-26 GWs multivariable } \\
\hline & & & Model I & Model II* & Model I & Model II * \\
\hline \multicolumn{7}{|l|}{ FABP4 } \\
\hline Q1: & 11 & 54 & 1.00 & 1.00 & 1.00 & 1.00 \\
\hline Q2: & 27 & 53 & 2.38 (1.05 to 5.43$)$ & 2.97 (1.17 to 7.53$)$ & $5.99(1.88$ to 19.1$)$ & 5.10 (1.37 to 19.0$)$ \\
\hline Q3: & 31 & 54 & 3.14 (1.36 to 7.22$)$ & 3.74 (1.46 to 9.57 ) & 7.42 (2.39 to 23.0$)$ & 8.35 (2.28 to 30.6 ) \\
\hline Q4: & 35 & 53 & 3.79 (1.63 to 8.85$)$ & 4.87 (1.84 to 12.9$)$ & 11.8 (3.57 to 38.9 ) & $11.0(2.85$ to 42.7$)$ \\
\hline$P$ for trend & & & 0.005 & $<0.0001$ & 0.004 & 0.001 \\
\hline \multicolumn{7}{|l|}{ Chemerin } \\
\hline Q1: & 9 & 53 & 1.00 & 1.00 & 1.00 & 1.00 \\
\hline Q2: & 33 & 53 & 3.81 (1.56 to 9.29$)$ & 3.62 (1.47 to 8.91$)$ & 1.59 (0.63 to 4.02$)$ & 1.26 (0.48 to 3.33 ) \\
\hline Q3: & 22 & 53 & 2.95 (1.14 to 7.59$)$ & 2.81 (1.07 to 7.36$)$ & 2.01 (0.77 to 5.21$)$ & 1.63 (0.59 to 4.53$)$ \\
\hline Q4: & 39 & 53 & 5.44 (2.05 to 14.4$)$ & 4.89 (1.83 to 13.1$)$ & 4.90 (1.97 to 12.2$)$ & $4.92(1.88$ to 12.9$)$ \\
\hline$P$ for trend & & & 0.003 & $<0.0001$ & 0.007 & $<0.0001$ \\
\hline \multicolumn{7}{|l|}{ IL-6 } \\
\hline Q1: & 6 & 41 & 1.00 & 1.00 & 1.00 & 1.00 \\
\hline Q2: & 16 & 40 & $3.26(1.06$ to 10.0$)$ & 3.74 (1.17 to 12.0$)$ & 1.62 (0.61 to 4.32$)$ & 1.75 (0.58 to 5.28 ) \\
\hline Q3: & 23 & 41 & 4.01 (1.40 to 11.5$)$ & 3.77 (1.31 to 10.9$)$ & 2.34 (0.89 to 6.13$)$ & 2.40 (0.81 to 7.08$)$ \\
\hline Q4: & 35 & 40 & 7.53 (2.53 to 22.4$)$ & 9.44 (2.92 to 30.5 ) & 3.31 (1.33 to 8.24$)$ & 3.44 (1.28 to 9.28 ) \\
\hline$P$ for trend & & & $<0.0001$ & 0.007 & $<0.001$ & 0.01 \\
\hline \multicolumn{7}{|l|}{ Leptin } \\
\hline Q1: & 18 & 54 & 1.00 & 1.00 & 1.00 & 1.00 \\
\hline Q2: & 10 & 53 & 0.65 (0.27 to 1.55$)$ & 0.60 (0.24 to 1.49$)$ & 0.83 (0.36 to 1.90$)$ & 0.85 (0.36 to 2.00 ) \\
\hline Q3: & 28 & 54 & 2.01 (0.90 to 4.49$)$ & 1.86 (0.80 to 4.31$)$ & 1.54 (0.67 to 3.56$)$ & 1.44 (0.59 to 3.50$)$ \\
\hline Q4: & 48 & 53 & 3.56 (1.66 to 7.64$)$ & 3.39 (1.52 to 7.55$)$ & 3.13 (1.43 to 6.88$)$ & 3.45 (1.43 to 8.32 ) \\
\hline$P$ for trend & & & $<0.0001$ & 0.001 & $<0.0001$ & 0.001 \\
\hline \multicolumn{7}{|l|}{ sOB-R } \\
\hline Q1: & 56 & 54 & 1.00 & 1.00 & 1.00 & 1.00 \\
\hline Q2: & 21 & 53 & 0.43 (0.22 to 0.81$)$ & 0.37 (0.19 to 0.74$)$ & 0.25 (0.12 to 0.55$)$ & 0.20 (0.09 to 0.48 ) \\
\hline Q3: & 14 & 54 & 0.31 (0.16 to 0.63$)$ & 0.28 (0.13 to 0.59 ) & 0.17 (0.07 to 0.40$)$ & 0.11 (0.04 to 0.31$)$ \\
\hline Q4: & 13 & 53 & 0.23 (0.11 to 0.50$)$ & 0.24 (0.11 to 0.52 ) & 0.15 (0.06 to 0.35$)$ & 0.14 (0.06 to 0.33 ) \\
\hline$P$ for trend & & & $<0.0001$ & $<0.0001$ & $<0.0001$ & $<0.0001$ \\
\hline \multicolumn{7}{|c|}{ Adiponectin } \\
\hline Q1: & 43 & 54 & 1.00 & 1.00 & 1.00 & 1.00 \\
\hline Q2: & 29 & 53 & 0.59 (0.31 to 1.14$)$ & 0.63 (0.32 to 1.25$)$ & $0.47(0.24$ to 0.92$)$ & 0.52 (0.25 to 1.07$)$ \\
\hline Q3: & 24 & 54 & 0.51 (0.25 to 1.03$)$ & 0.45 (0.22 to 0.95$)$ & 0.26 (0.12 to 0.57$)$ & 0.26 (0.11 to 0.59 ) \\
\hline Q4: & 8 & 53 & 0.14 (0.05 to 0.34$)$ & 0.13 (0.05 to 0.35$)$ & 0.17 (0.07 to 0.42$)$ & 0.15 (0.06 to 0.40$)$ \\
\hline$P$ for trend & & & $<0.0001$ & $<0.0001$ & $<0.0001$ & $<0.0001$ \\
\hline \multicolumn{7}{|c|}{ HMW-adiponectin } \\
\hline Q1: & 45 & 54 & 1.00 & 1.00 & 1.00 & 1.00 \\
\hline Q2: & 30 & 53 & 0.59 (0.31 to 1.12$)$ & 0.56 (0.28 to 1.08$)$ & $0.59(0.30$ to 1.17$)$ & 0.71 (0.34 to 1.51$)$ \\
\hline Q3: & 18 & 54 & 0.34 (0.16 to 0.70$)$ & 0.31 (0.14 to 0.66 ) & $0.26(0.12$ to 0.55$)$ & 0.25 (0.11 to 0.58 ) \\
\hline Q4: & 11 & 53 & 0.20 (0.09 to 0.46$)$ & 0.20 (0.08 to 0.48$)$ & 0.20 (0.08 to 0.48$)$ & 0.20 (0.08 to 0.50$)$ \\
\hline$P$ for trend & & & $<0.0001$ & $<0.0001$ & $<0.0001$ & $<0.0001$ \\
\hline \multicolumn{7}{|l|}{ Omentin-1 } \\
\hline Q1: & 24 & 54 & 1.00 & 1.00 & 1.00 & 1.00 \\
\hline
\end{tabular}


Table 2 Continued

\begin{tabular}{|c|c|c|c|c|c|c|}
\hline & \multirow{2}{*}{$\begin{array}{l}\text { GDM } \\
\text { cases (n) }\end{array}$} & \multirow{2}{*}{$\begin{array}{l}\text { Controls } \\
\text { (n) }\end{array}$} & \multicolumn{2}{|c|}{ 10-14 GWs multivariable } & \multicolumn{2}{|c|}{ 15-26 GWs multivariable } \\
\hline & & & Model I & Model II* & Model I & Model II * \\
\hline Q2: & 37 & 53 & 1.63 (0.79 to 3.35$)$ & 1.69 (0.80 to 3.58$)$ & 1.25 (0.63 to 2.45$)$ & 1.39 (0.69 to 2.79$)$ \\
\hline Q3: & 19 & 54 & 0.71 (0.32 to 1.57$)$ & 0.77 (0.33 to 1.79$)$ & $0.71(0.33$ to 1.53$)$ & $0.89(0.40$ to 2.01$)$ \\
\hline Q4: & 24 & 53 & $1.00(0.44$ to 2.25$)$ & 0.95 (0.40 to 2.26$)$ & $0.80(0.36$ to 1.77$)$ & $0.80(0.35$ to 1.87$)$ \\
\hline$P$ for trend & & & 0.47 & 0.37 & 0.44 & 0.44 \\
\hline \multicolumn{7}{|l|}{ Vaspin } \\
\hline Q1: & 19 & 52 & 1.00 & 1.00 & 1.00 & 1.00 \\
\hline Q2: & 22 & 52 & $1.25(0.58$ to 2.71$)$ & $1.66(0.72$ to 3.81$)$ & 1.26 (0.59 to 2.67$)$ & 1.17 (0.52 to 2.62$)$ \\
\hline Q3: & 39 & 52 & $2.13(1.04$ to 4.37$)$ & 2.37 (1.09 to 5.16$)$ & 1.49 (0.73 to 3.05$)$ & 1.67 (0.78 to 3.55$)$ \\
\hline Q4: & 22 & 51 & 1.21 (0.58 to 2.52$)$ & 1.50 (0.70 to 3.22$)$ & 1.63 (0.78 to 3.39$)$ & $1.92(0.87$ to 4.26$)$ \\
\hline$P$ for trend & & & 0.96 & 0.23 & 0.71 & 0.09 \\
\hline \multicolumn{7}{|l|}{ RBP4 } \\
\hline Q1: & 22 & 54 & 1.00 & 1.00 & 1.00 & 1.00 \\
\hline Q2: & 27 & 53 & 1.27 (0.64 to 2.55$)$ & 1.31 (0.63 to 2.69$)$ & 1.09 (0.54 to 2.22$)$ & 1.29 (0.60 to 2.79$)$ \\
\hline Q3: & 22 & 54 & 1.00 (0.48 to 2.10$)$ & $1.13(0.52$ to 2.45$)$ & 1.14 (0.55 to 2.36$)$ & 1.28 (0.58 to 2.83$)$ \\
\hline Q4: & 33 & 53 & 1.54 (0.78 to 3.03$)$ & 1.69 (0.83 to 3.45$)$ & 0.85 (0.40 to 1.80$)$ & 0.99 (0.44 to 2.28$)$ \\
\hline$P$ for trend & & & 0.28 & 0.65 & 0.18 & 0.91 \\
\hline
\end{tabular}

Model I adjusted for matching factors matched within a range (maternal age and gestational week of blood collection).

*Model II further adjusted for parity, family history of diabetes, and parity.

FABP4, fatty acid binding protein-4; GDM, gestational diabetes mellitus; GW, gestational week; HMW-adiponectin, high-molecular weight-adiponectin; IL-6, interleukin-6; Q, quartile; RBP4, retinol binding protein-4; sOB-R, soluble leptin receptor.

insulin, and hsCRP, suggesting a role of FABP4 in glucoseinsulin homeostasis and inflammatory pathways which may contribute to the subsequent risk of GDM.

Chemerin is a chemoattractant protein implicated in regulating adipogenesis and lipolysis and promotes the migration of a variety of immune cell types. ${ }^{34}$ In the current study, higher concentrations of chemerin in early and mid-pregnancy were significantly associated with increased GDM risk, and throughout pregnancy levels were consistently higher among women who developed GDM compared with controls. To date, most studies of chemerin and GDM have been cross-sectional, with limited data from prospective studies. $^{79} 25$ One study among native Scandinavians reported higher levels of chemerin at four time points throughout pregnancy (14-38 GWs) among women who developed GDM, ${ }^{7}$ although differences were not statistically significant. The relatively small number of GDM cases $(n=48)$ in the previous study may have impeded their

Table 3 Spearman partial correlations among controls: adipokine concentrations at 10-14 GWs with glucose metabolism and cardiometabolic markers at 15-26 GWs

\begin{tabular}{|c|c|c|c|c|c|c|c|c|}
\hline & HOMA-IR & Glucose & Insulin & C-peptide & hsCRP & HDLD & LDLD & TGs \\
\hline FABP4 & 0.16 & $0.25^{\star \star}$ & $0.17^{*}$ & $0.27^{\star \star}$ & $0.32^{\star \star \star}$ & $-0.24^{\star \star}$ & 0.05 & 0.13 \\
\hline Chemerin & 0.02 & 0.09 & 0.03 & 0.10 & $0.26^{\star \star}$ & -0.05 & 0.08 & $0.17^{\star}$ \\
\hline IL-6 & $0.30^{\star \star \star}$ & 0.08 & $0.31^{\star \star *}$ & $0.29^{\star \star \star}$ & $0.16^{\star}$ & 0.08 & -0.02 & -0.02 \\
\hline Leptin & $0.30^{\star \star \star}$ & $0.22^{\star \star}$ & $0.33^{\star \star \star}$ & $0.39^{\star \star \star}$ & $0.48^{\star \star \star}$ & -0.13 & 0.10 & 0.07 \\
\hline sOB-R & $-0.27^{\star \star \star}$ & $-0.27^{\star \star}$ & $-0.30^{\star \star \star}$ & $-0.36^{\star \star \star}$ & $-0.24^{\star \star}$ & $0.25^{\star \star}$ & 0.09 & -0.13 \\
\hline Adiponectin & $-0.22^{\star \star}$ & $-0.29^{\star \star \star}$ & $-0.24^{\star \star}$ & $-0.28^{\star \star}$ & -0.12 & $0.36^{\star \star \star}$ & -0.01 & $-0.27^{\star \star \star}$ \\
\hline HMW-adiponectin & $-0.23^{\star \star}$ & $-0.31^{\star \star *}$ & $-0.24^{\star \star}$ & $-0.29^{\star \star}$ & -0.11 & $0.34^{\star * \star}$ & -0.01 & $-0.30^{\star *}$ \\
\hline Omentin-1 & $-0.19^{\star}$ & -0.06 & $-0.21^{*}$ & $-0.21^{\star}$ & -0.01 & 0.13 & 0.00 & $-0.14^{*}$ \\
\hline Vaspin & 0.03 & 0.10 & 0.03 & 0.03 & -0.07 & -0.12 & 0.03 & 0.07 \\
\hline RBP4 & 0.00 & 0.07 & 0.00 & 0.07 & -0.08 & -0.02 & $0.14^{*}$ & $0.25^{\star \star}$ \\
\hline
\end{tabular}

${ }^{*} \mathrm{P}<0.05 ;{ }^{* *} \mathrm{p}<0.01 ;{ }^{* \star} \mathrm{p}<0.001$; adjusts for age, GW of blood collection, nulliparity, and family history of diabetes.

FABP4, fatty acid binding protein-4; GW, gestational week; HDLD, high-density lipoprotein; HMW-adiponectin, high-molecular weight-

adiponectin; HOMA-IR, homeostatic model assessment of insulin resistance; hsCRP, high-sensitivity C-reactive protein; IL-6, interleukin-6;

LDLD, low-density lipoprotein; RBP4, retinol binding protein-4; sOB-R, soluble leptin receptor; TGs, triglycerides. 
ability to detect statistical differences in chemerin levels, which were observed in our study with a larger number of GDM cases.

Our observed positive association between chemerin and GDM is biological plausible, although the precise mechanistic role is yet to be discovered. Data indicate that chemerin has both anti-inflammatory and proinflammatory properties, and has been correlated with IL- 6 and tumour necrosis factor alpha (TNF $\alpha),{ }^{3}$ both of which suppress insulin receptor substrate-1 (IRS1). ${ }^{35}$ Chemerin regulates adipogenesis, with increased expression in adipose tissue resulting in insulin resistance in adipocytes. ${ }^{34}$ In our data, chemerin was correlated with triglycerides and hsCRP, which align with previous data implicating disruptions in lipid metabolism and inflammation in the pathogenesis of GDM. $^{4}$

IL-6 is a traditional cytokine involved in inflammatory responses. ${ }^{35}$ We observed that higher IL-6 concentrations in early and mid-pregnancy were associated with GDM risk, and throughout pregnancy levels were consistently higher among women who developed GDM compared with controls. Prospective studies of IL-6 and GDM in early pregnancy are sparse and have included a limited number of GDM cases $(n=14-40)$. Two prior studies have reported significantly higher IL-6 concentrations at 11-15 GWs among women who subsequently developed GDM. ${ }^{36} 37$ However, in other small studies with less than 30 GDM cases the higher IL-6 concentrations among GDM cases were not statistically significant. ${ }^{38-40}$ Our data provide the first report of longitudinal profiles of IL-6 throughout pregnancy by GDM status among a larger racially/ethnically diverse sample.

Interestingly, we observed that the association of IL-6 at 15-26 GWs and GDM was attenuated after adjustment for prepregnancy BMI indicating that during this phase of pregnancy other tissues, such as placenta, may be the primary source of IL-6. This finding is consistent with a prior null study of IL-6 measured at roughly the twentieth week of pregnancy and GDM risk which adjusted for prepregnancy BMI. ${ }^{41}$ IL-6 directly inhibits insulin sensitivity by inhibiting tyrosine phosphorylation of IRS1, and it stimulates the release of other hormones that contribute to insulin resistance.$^{35}$ In the current study, IL-6 was positively correlated with subsequent insulin. It is plausible that IL-6 suppression of IRS1 results in higher circulating insulin but lower sensitivity to insulin action and thus the positive association with GDM risk.

sOB-R is the primary binding protein for leptin. ${ }^{42}$ The current study is the first to longitudinally examine sOB-R profiles throughout pregnancy by GDM status. Consistently lower levels were observed among GDM cases. We found that in early and mid-pregnancy sOB-R was associated with a roughly $75 \%$ lower likelihood of developing GDM, whereas higher leptin was associated with increased GDM risk. Studies on sOB-R and GDM are limited, with only one prospective study in early pregnancy. Consistent with our findings they reported an inverse association between sOB-R and GDM risk. ${ }^{15}$ Most previously reviewed studies ${ }^{5}$ reported that higher leptin was associated with increased GDM risk, although three studies reported no associations. $^{254344}$

Previous data indicate that low concentrations of sOB-R reflect reduced leptin sensitivity and membrane expression of the leptin receptors. ${ }^{42}$ We observed that higher leptin, and lower sOB-R concentrations were associated with greater GDM risk which may represent resistance to leptin. Animal and epidemiological studies indicate that leptin resistance and impaired leptin signaling in energy homeostasis impacts insulin resistance through decreasing insulin sensitivity in adipose tissue and skeletal muscle, ${ }^{45}$ and by upregulating acute phase inflammatory markers. ${ }^{46}$ In the current study, leptin was positively correlated with markers of glucose metabolism and inflammation, which aligns with previous data implicating leptin in these pathways.

In the current study, we confirmed the association between reduced adiponectin in early to mid-pregnancy and GDM risk. ${ }^{5}$ Adiponectin has different binding affinities, and HMW-adiponectin is thought to represent the most active isoform of adiponectin and account for most of the insulin-sensitizing effects. ${ }^{47}$ Despite this, we found that the strength of the associations with reduced GDM risk were similar for adiponectin and HMW-adiponectin. Adiponectin stimulates glucose uptake in skeletal muscle and reduces hepatic glucose production through activating AMP-activated protein kinase, and therefore is an insulinsensitizing hormone. ${ }^{48}$ Proinflammatory cytokines such as TNF- $\alpha$ and IL- 6 suppress the transcription of adiponectin in adipocytes, ${ }^{49}$ which has been suggested to explain the lower levels of serum adiponectin in GDM. ${ }^{50}$

Our study has several unique strengths. First, the prospective and longitudinal data collection allowed us to examine the temporal association of adipokine levels in early and mid-pregnancy with subsequent development of GDM. Second, with the measurement of adipokines four times throughout pregnancy, we had the unique ability to profile the physiologic trends of a comprehensive panel of adipokines throughout pregnancy while distinguishing between women with and without GDM. Third, the longitudinal measures of glucose metabolic markers and the estimated correlations with adipokines were analyzed and provided insight into the interpretation of observed associations between adipokines and GDM. Lastly, the current study examined a panel of adipokines (some of which have been underexplored; FABP4, chemerin, sOB-R, omentin-1, vaspin, and RBP4), which provided insight to the value of novel markers of adipose tissue function and inflammation in GDM. The inclusion of multiple adipokines such as FABP4, chemerin, sOB-R, and HMW-adiponectin at 10-14 GWs and 15-26 GWs improved the prediction of GDM over conventional risk factors resulting in a significant increase in the AUC. Our study also explored the combined influence of this panel of adipokines, based on an adipokine score, on GDM risk. The use of an adipokine score which represents the amount of high-risk adipokine levels within an individual, captures both the magnitude 
of the association with GDM risk and at least some of the complexity by which these adipokines are related. The OR for the association was significant even after adjusting for other risk factors. However, the $95 \%$ CI of the OR was wide and therefore we suggest caution in regard to the precision of the estimate.

Several limitations to our data should be considered. Although our sample was drawn from a larger cohort, the number of participants in the current analysis (107 GDM cases and 214 non-GDM controls) prevented us from creating training and validation data sets. Despite this, we used leave-one-out cross-validation to minimize model overfitting. The NICHD Fetal Growth Studies inclusion criteria were selected to create a sample of participants without major chronic conditions and therefore may not be generalizable to high-risk populations; nevertheless, our study is one of the first to examine these adipokines among participants from a diverse racial/ethnic background which increases the generalizability of our findings. Although we have controlled for known major confounders, similar to other observational studies these do not represent causal effects, and we cannot completely exclude the possibility for residual confounding by unmeasured factors or measurement errors.

In summary, in the current study, a panel of adipokines as early as $10-14$ GWs, which is approximately $10-18$ weeks earlier than when GDM is typically screened for, were significantly related to GDM risk. The novel findings on lower levels of sOB-R and higher levels of FABP4 and chemerin in both first and second trimesters in association with an elevated risk of subsequent GDM suggest a pathophysiologic role of these adipokines in GDM etiology. Future studies are warranted to investigate the underlying molecular mechanisms.

\section{Author affiliations}

Colorado School of Public Health, University of Colorado Denver - Anschutz Medical Campus, Aurora, Colorado, USA

${ }^{2}$ Division of Intramural Population Health Research, Eunice Kennedy Shriver National Institute of Child Health and Human Development, Bethesda, Maryland, USA

${ }^{3}$ Biostatistics, University of Pennsylvania Perelman School of Medicine,

Philadelphia, Pennsylvania, USA

${ }^{4}$ Glotech, Rockville, Maryland, USA

${ }^{5}$ Division of Research, Kaiser Permanente Northern California, Oakland, California, USA

${ }^{6}$ Department of Epidemiology and Biostatistics, University of California San Francisco, San Francisco, California, USA

${ }^{7}$ Cardiovascular Branch, National Heart Lung and Blood Institute, Bethesda, Maryland, USA

${ }^{8}$ Department of Public Health Sciences, Clemson University College of Behavioral, Social and Health Sciences, Clemson, South Carolina, USA

${ }^{9}$ Laboratory Medicine and Pathology, University of Minnesota System, Minneapolis, Minnesota, USA

${ }^{10}$ Epidemiology, University of California Los Angeles Jonathan and Karin Fielding School of Public Health, Los Angeles, California, USA

Correction notice This article has been corrected since it was published. Author name corrected from Hiaming Cao to Haiming Cao.

Acknowledgements The authors thank all research teams at each participating clinical center for their efforts, including Christina Care Health Systems; University of California, Irvine; Long Beach Memorial Medical Center; Northwestern University;
Medical University of South Carolina; Columbia University; New York Hospital Queens; St Peters' University Hospital; University of Alabama at Birmingham; Women and Infants Hospital of Rhode Island; Fountain Valley Regional Hospital and Medical Center; and Tufts University. The authors also thank the C-TASC Corporation and Emmes Corporation for providing data and image coordination and the Department of Laboratory Medicine and Pathology, University of Minnesota that provided laboratory resources essential to test blood samples for biomarkers.

Contributors ECF analyzed data and wrote the first draft of the manuscript. ML, SNH, YC, JC, JiW contributed to data analysis, statistical approach, and reviewed the manuscript. ECF contributed to study coordination, data interpretation, and manuscript reviewing. ECF, ML, SNH, HC, KK, LR, JoW, and LC contributed to data interpretation and reviewed the manuscript. MYT contributed to laboratory testing and reviewed the manuscript. CZ obtained funding, designed and oversaw the study and revised the manuscript. All authors contributed to the interpretation of the results and revision of the manuscript for important intellectual content and approved the final version of the manuscript. ECF and CZ are the guarantors of this work and, as such, had full access to all the data in the study and take responsibility for the integrity of the data and the accuracy of the data analysis.

Funding This research was supported by the Eunice Kennedy Shriver National Institute of Child Health and Human Development intramural funding and included American Recovery and Reinvestment Act funding via contract numbers HHSN275200800013C, HHSN2752008000021, HHSN27500006, HHSN275200800003IC, HHSN275200800014C, HHSN275200800012C, HHSN275200800028C, HHSN275201000009C, and HHSN275201000001Z. YZ was supported by the National Institute of Diabetes and Digestive and Kidney Diseases (grant number K01DK120807). ECF is a participant in the NIH Graduate Partnership Program and a graduate student at Clemson University.

Competing interests None declared.

\section{Patient consent for publication Not required.}

Ethics approval This study was carried out following the rules of the Declaration of Helsinki. Institutional review board approval was obtained for all the participating clinical sites (Christiana Care Health System, Columbia University, Fountain Valley, Long Beach Memorial Medical Center, Medical University of South Carolina, New York Hospital Queens, Northwestern University, St. Peter's University Hospital, Tufts University, University of Alabama at Birmingham, University of California, Irvine, Women and Infants Hospital of Rhode Island), data coordinating centers (Clinical Trials \& Surveys Corporation and the Emmes Corporation), and NICHD (approval number: 09-CH-N152). All participants provided written, informed consent.

Provenance and peer review Not commissioned; externally peer reviewed.

Data availability statement Data are available upon reasonable request. The data, along with a set of guidelines for researchers applying for the data, will be posted to a data-sharing site, the NICHD/DIPHR Biospecimen Repository Access and Data Sharing (https://brads.nichd.nih.gov) (BRADS).

Open access This is an open access article distributed in accordance with the Creative Commons Attribution Non Commercial (CC BY-NC 4.0) license, which permits others to distribute, remix, adapt, build upon this work non-commercially, and license their derivative works on different terms, provided the original work is properly cited, appropriate credit is given, any changes made indicated, and the use is non-commercial. See: http://creativecommons.org/licenses/by-nc/4.0/.

ORCID iDs

Ellen C Francis http://orcid.org/0000-0001-8618-045X

Mengying Li http://orcid.org/0000-0002-3713-1683

\section{REFERENCES}

1 Metzger BE, Coustan DR. Summary and recommendations of the fourth International Workshop-Conference on gestational diabetes mellitus. The organizing Committee. Diabetes Care 1998;21 Suppl 2:B161-7

2 Sermer M, Naylor CD, Gare DJ, et al. Impact of increasing carbohydrate intolerance on maternal-fetal outcomes in 3637 women without gestational diabetes. The Toronto Tri-Hospital gestational diabetes project. Am J Obstet Gynecol 1995;173:146-56.

3 Blüher M. Importance of adipokines in glucose homeostasis. Diabetes Manag 2013;3:389-400.

4 Pantham P, Aye ILMH, Powell TL. Inflammation in maternal obesity and gestational diabetes mellitus. Placenta 2015;36:709-15

5 Bao W, Baecker A, Song Y, et al. Adipokine levels during the first or early second trimester of pregnancy and subsequent risk of 
gestational diabetes mellitus: a systematic review. Metabolism 2015;64:756-64.

6 Fasshauer M, Blüher M, Stumvoll M. Adipokines in gestational diabetes. Lancet Diabetes Endocrinol 2014;2:488-99.

7 Ueland T, Michelsen AE, Aukrust P, et al. Adipokines and macrophage markers during pregnancy-Possible role for SCD163 in prediction and progression of gestational diabetes mellitus. Diabetes Metab Res Rev 2019;35:e3114.

8 Tu W-J, Guo M, Shi X-D, et al. First-Trimester serum fatty acidbinding protein 4 and subsequent gestational diabetes mellitus. Obstet Gynecol 2017;130:1011-6.

9 Yang X, Quan X, Lan Y, et al. Serum chemerin level during the first trimester of pregnancy and the risk of gestational diabetes mellitus. Gynecol Endocrinol 2017;33:770-3.

10 Hou W, Meng X, Zhao A, et al. Development of multimarker diagnostic models from metabolomics analysis for gestational diabetes mellitus (GDM). Mol Cell Proteomics 2018;17:431-41.

11 Nanda S, Nikoletakis G, Markova D, et al. Maternal serum retinol-binding protein-4 at 11-13 weeks' gestation in normal and pathological pregnancies. Metabolism 2013;62:814-9.

12 Ping $\mathrm{F}$, Xiang $\mathrm{H}$-ding, Li M, et al. Effects of variation in retinol binding protein 4 gene and adipose specific expression of gestational diabetes in Beijing, China. Diabetes Res Clin Pract 2012;97:283-9.

13 Abetew DF, Qiu C, Fida NG, et al. Association of retinol binding protein 4 with risk of gestational diabetes. Diabetes Res Clin Pract 2013;99:48-53.

14 Yuan X-S, Shi H, Wang H-Y, et al. Ficolin-3/adiponectin ratio for the prediction of gestational diabetes mellitus in pregnant women. $J$ Diabetes Investig 2018;9:403-10.

15 Sommer C, Gulseth HL, Jenum AK, et al. Soluble leptin receptor and risk of gestational diabetes in a multiethnic population: a prospective cohort study. J Clin Endocrinol Metab 2016;101:4070-5.

16 Grewal J, Grantz KL, Zhang C, et al. Cohort profile: NICHD fetal growth Studies-Singletons and twins. Int J Epidemiol 2018;47:25-25l.

17 Zhu Y, Mendola P, Albert PS, et al. Insulin-Like growth factor axis and gestational diabetes mellitus: a longitudinal study in a Multiracial cohort. Diabetes 2016;65:3495-504.

18 Committee on Practice Bulletins-Obstetrics. ACOG practice Bulletin No. 190: gestational diabetes mellitus. Obstet Gynecol 2018;131:e49-64.

19 American College of Obstetricians and Gynecologists Committee on Practice Bulletins--Obstetrics. ACOG practice Bulletin. clinical management guidelines for obstetrician-gynecologists. number 30, September 2001 (replaces technical Bulletin number 200, December 1994). gestational diabetes. Obstet Gynecol 2001;98:525-38.

20 Zhang C, Hediger ML, Albert PS, et al. Association of maternal obesity with longitudinal ultrasonographic measures of fetal growth: findings from the NICHD fetal growth Studies-Singletons. JAMA Pediatr 2018;172:24-31.

21 Camhi SM, Bray GA, Bouchard C, et al. The relationship of waist circumference and BMI to visceral, subcutaneous, and total body fat: sex and race differences. Obesity 2011;19:402-8.

22 American Diabetes Association. Diagnosis and classification of diabetes mellitus. Diabetes Care 2010;33 Suppl 1:S62-9.

23 Hertzmark E, Li R, Hong B, et al. The SAS GLMCURV9 macro. Harvard University, 2014.

24 Matthews DR, Hosker JP, Rudenski AS, et al. Homeostasis model assessment: insulin resistance and ?-cell function from fasting plasma glucose and insulin concentrations in man. Diabetologia 1985;28:412-9.

25 Guelfi KJ, Ong MJ, Li S, et al. Maternal circulating adipokine profile and insulin resistance in women at high risk of developing gestational diabetes mellitus. Metabolism 2017;75:54-60.

26 Cao. Semi-parametric efficient prediction of binary outcomes when some predictors are incomplete via Post-stratification;2019. (under review).
27 Breslow NE, Chatterjee N. Design and analysis of two-phase studies with binary outcome applied to Wilms tumour prognosis. Journal of the Royal Statistical Society: Series C 1999;48:457-68.

28 Harrell FE. Regression modeling strategies:with applications to linear models, logistic and ordinal regression, and survival analysis. Springer, 2015.

29 Efron B, Tibshirani RJ. An introduction to the bootstrap. New York: Chapman \& Hall, 1993.

30 Donovan BM, Breheny PJ, Robinson JG, et al. Development and validation of a clinical model for preconception and early pregnancy risk prediction of gestational diabetes mellitus in nulliparous women. PLoS One 2019;14:e0215173.

31 Cao H, Sekiya M, Ertunc ME, et al. Adipocyte lipid chaperone AP2 is a secreted adipokine regulating hepatic glucose production. Cell Metab 2013;17:768-78.

32 Bellos I, Fitrou G, Pergialiotis V, et al. Serum levels of adipokines in gestational diabetes: a systematic review. J Endocrinol Invest 2019;42:621-31.

33 Hotamisligil GS, Bernlohr DA. Metabolic functions of FABPs-mechanisms and therapeutic implications. Nat Rev Endocrinol 2015;11:592-605.

34 Roman AA, Parlee SD, Sinal CJ. Chemerin: a potential endocrine link between obesity and type 2 diabetes. Endocrine 2012:42:243-51.

35 Senn JJ, Klover PJ, Nowak IA, et al. Interleukin-6 induces cellular insulin resistance in hepatocytes. Diabetes 2002;51:3391-9.

36 Abell SK, Shorakae S, Harrison CL, et al. The association between dysregulated adipocytokines in early pregnancy and development of gestational diabetes. Diabetes Metab Res Rev 2017;33:dmrr.2926.

37 Hassiakos D, Eleftheriades M, Papastefanou I, et al. Increased maternal serum interleukin- 6 concentrations at 11 to 14 weeks of gestation in low risk pregnancies complicated with gestational diabetes mellitus: development of a prediction model. Horm Metab Res 2016;48:35-41.

38 Lain KY, Daftary AR, Ness RB, et al. First trimester adipocytokine concentrations and risk of developing gestational diabetes later in pregnancy. Clin Endocrinol 2008;69:407-11.

39 Georgiou HM, Lappas M, Georgiou GM, et al. Screening for biomarkers predictive of gestational diabetes mellitus. Acta Diabetol 2008;45:157-65

40 Dasanayake AP, Chhun N, Tanner ACR, et al. Periodontal pathogens and gestational diabetes mellitus. J Dent Res 2008;87:328-33.

41 Bossick AS, Peters RM, Burmeister $C$, et al. Antenatal inflammation and gestational diabetes mellitus risk among pregnant AfricanAmerican women. J Reprod Immunol 2016;115:1-5.

42 Schaab M, Kratzsch J. The soluble leptin receptor. Best Pract Res Clin Endocrinol Metab 2015;29:661-70.

43 Maitland RA, Seed PT, Briley AL, et al. Prediction of gestational diabetes in obese pregnant women from the UK pregnancies better eating and activity (UPBEAT) pilot trial. Diabet Med 2014;31:963-70.

44 Fruscalzo A, Londero AP, Biasizzo J, et al. Second trimester maternal plasma and amniotic fluid adipokines in women who will develop gestational diabetes mellitus. Gynecol Endocrinol 2015;31:934-8.

45 Rehman K, Akash MSH, Alina Z. Leptin: a new therapeutic target for treatment of diabetes mellitus. J Cell Biochem 2018;119:5016-27.

46 Pradhan ADet al. C-Reactive protein, interleukin 6, and risk of developing type 2 diabetes mellitus. JAMA 2001;286:327-34.

47 Wang Y, Lam KSL, Yau M-hon, et al. Post-Translational modifications of adiponectin: mechanisms and functional implications. Biochem $J$ 2008;409:623-33.

48 Nigro E, Scudiero O, Monaco ML, et al. New insight into adiponectin role in obesity and obesity-related diseases. Biomed Res Int 2014;2014:1-14.

49 Fantuzzi G. Adipose tissue, adipokines, and inflammation. J Allergy Clin Immunol 2005;115:911-9.

50 Barbour LA, McCurdy CE, Hernandez TL, et al. Cellular mechanisms for insulin resistance in normal pregnancy and gestational diabetes. Diabetes Care 2007;30 Suppl 2:S112-9. 